\title{
2012 Cold Spring Harbor Asia Conference on Synthetic Biology
}

\author{
Hongyu Zhao* \\ Department of Biological Sciences and Biotechnology, School of Life Sciences, Tsinghua University, Beijing 100084, China \\ * Correspondence: zhaohongyu@973pha.org
}

Received January 1, 2013

Cold Spring Harbor Asia Conference on Synthetic Biology was held at the Suzhou Dushu Lake Conference Center, China, on Nov 26-30, 2012. The conference was organized by George Guo-Qiang Chen (Tsinghua University, China), Sang Yup Lee (Korea Advanced Institute of Science and Technology, Korea), Birger Lindberg Møller (University of Copenhagen, Denmark) and Hiroki Ueda (RIKEN Kobe Institute, Japan).

Synthetic Biology has been making rapid progresses despite of new challenges. In the opening remark, George Guo-Qiang Chen (Tsinghua University, China) briefly introduced the development of Synthetic Biology in the past. He pointed out that many nations have listed Synthetic Biology as one of the key technologies for the national economic development. Then he shared his views of perspectives and challenges in the field of Synthetic Biology.

The conference included three keynote speeches, delivered by Birger Lindberg Møller from University of Copenhagen in Denmark, Lars K. Nielsen from the University of Queensland in Australia and Sven Panke from ETH Zurich in Switzerland. Fifty-four oral presentations and forty-six poster presentations were selected from the openly submitted abstracts and arranged into eight sessions.

\section{Keynote speeches}

In the first keynote speech, Birger Lindberg Møller presented a lecture on "Plant power: the ultimate way to go green". Plant can produce bioactive defense compounds when attacked by insects and microbes. Several of these compounds are used in the treatment of human diseases including cancer. They use the "share-your-parts" principle of synthetic biology to promote the production of bioactive compounds that are difficult or impossible to synthesize using chemical methods. The second keynote speaker, Lars K. Nielsen presented "Metabolic and regulatory models for synthetic biology design". He reviewed the status of rational in-silico strain design, providing a cost effective way of constructing metabolic pathways for making biocompounds. The last keynote speaker Sven Panke provided promises and ambition on technical advances as well as on a conceptual rethinking of how we design biocatalysts. He illustrated principles of designing biocatalysts with examples from his own work on nanoand pico-liter reactor design for screening purposes, on real-time mass spectrometry for pathway analysis and optimization, and protein switching for orthogonal in vitro pathways.

\section{Prokaryotic genome engineering}

Yu-Sin Jang (KAIST, Korea) reported that the direct butanol-forming pathway is a better channel for optimization of butanol production. An interesting topic was shared by Akio Kawahara-Kobayash (Tokyo Institute of Technology, Japan). At earlier stages in the evolution of the universal genetic code, fewer than 20 amino acids were considered for use. They made artificial genetic codes involving a reduced alphabet to provide not only new insights into primordial genetic codes, but also an essential protein engineering tool for the assessment of the early stages of protein evolution and for the improvement of pharmaceuticals. To meet the demands of industrial production, microbes should maintain a maximized and fixed carbon flux towards target metabolites regardless of fluctuations in intracellular or extracellular environments. Yin Li (Institute of Microbiology, 
Chinese Academy of Sciences, China) showed how synthetic biology can be used to improve the robustness of industrial microbes through engineering of dynamic controllability, modular and hierarchical organization.

\section{Genome engineering of eukaryotic systems}

Ulrik Gether (University of Copenhagen, Denmark) presented the molecular function and perspectives of neurotransmitter sodium symporters (NSS) for synthetic biology. Kana Ishimatsu (Department of Computational Intelligence and Systems Science, Tokohama, Japan) reported that a synthetic gene network can be used to enable arbitrary control of cell-type ratio. Jin-Soo Kim (Seoul National University, Korea) told us the newest development about Zinc finger nucleases (ZFNs) and transcription activator-like effector nucleases (TALENs) for precise genome editing in cells and animals. Thomas Vogl (Graz University of Technology, Graz, Austria) reported synthetic promoters in the yeast Pichiapastoris for heterologous protein production. Yi Yang (East China University of Science and Technology, Shanghai, China) presented the light-switchable gene expression systems in eukaryotic and prokaryotic cells. Sun Chang Kim (KAIST, Daejeon, Korea) demonstrated a "top down" approach for minimizing the E. coli genome to create an artificial organism with "core" elements for free selfsustaining and self-replicating cells. Hal Alper (University of Texas at Austin, Austin, USA) lectured on recent advances in synthetic control of transcription by hybrid promoters in both Saccharomyces cerevisiae and the nonconventional yeast, Yarrowialipolytica.

\section{Designing cellular circuits}

Hiroki R. Ueda (RIKEN, Kobe, Japan) presented the systems and synthetic biology of biological timings. Qi Ouyang (Peking University, Beijing, China) discussed engineering of synthetic biological network. A lecture on how cells respire using synthetic biology was given by Caroline M. Aji-Franklin (Lawrence Berkeley National Laboratory, Berkeley, USA). Modification of the RpoS network with an artificial small RNA was presented by Ye Jin (GIAT, Guangzhou, China). Mario A. Marchisio (ETH Zurich, Basel, Switzerland) conducted the computational design and in vivo implement of Boolean gates.

\section{Metabolic engineering for chemicals, fuels and materials}

George Guo-Qiang Chen (Tsinghua University, Beijing, China) reported the progress on genomic manipulation for microbial production of diverse polyesters. Brian F. Pfleger (University of WisconsinMadison. Madison, USA) showed metabolic engineering of bacteria for production of specialty chemicals. Henrik Vibe Scheller (University of California, Berkeley, USA) presented engineering of plants for use as Biorefinery feed stocks. Genetic design of bacterial inclusion bodies as emerging drug delivery platforms was reported by Antonio Villaverde (University Autonoma de Barcelona, Barcelona, Spain). Oliver Yu (Wuxi New Way Biotechnology Ltd, Wuxi, China) demonstrated microbial fermentation of styrene as an industrial monomer. Huimin Zhao (University of Illinois

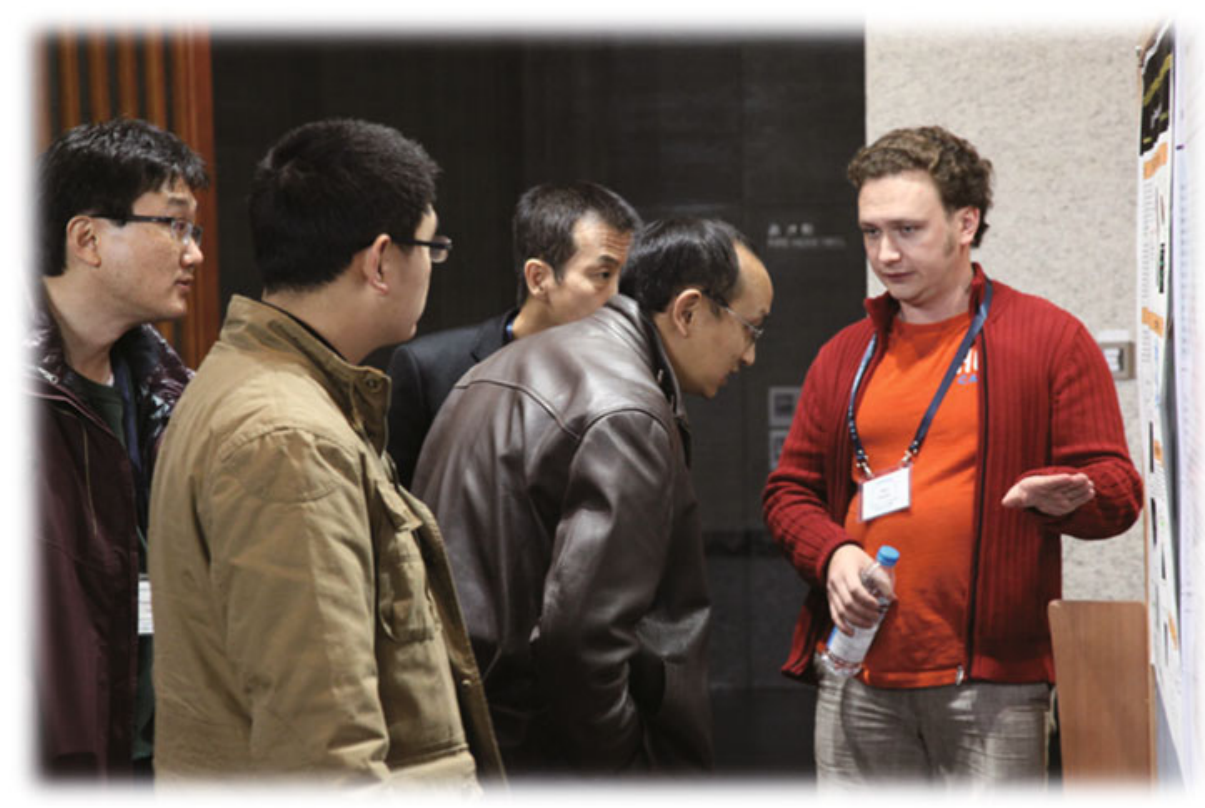

A photo taken in the poster section 
at Urbana-Champaign, Urbana, USA) presented a new tool for rapid construction of large DNA molecules and its applications, as well as a new tool for targeted genome engineering in mammalian systems. Dimitrios Stamou (University of Copenhagen. Denmark) showed the progress on lipid membrane nanotechnology for synthetic biology applications. Yanyan Li (Jiangnan University, Wuxi, China) reported that temperture-regulated membrane remodeling is essential for Francisella pathogenesis. Ryoji Sekine (Tokyo Institute of Technology, Yokohama-shi, Japan) talked about the progress on a repeatable diversification system for sustainable material production.

\section{Poster awards}

During the dinner of the last day, one Gold, two Silver and three Bronze awards in the poster section were selected. Haoqian Zhang (Peking University, Beijing, China) won the Gold Medal for his work on construction of a gene network that performs Pavlovian conditioning by a module-based and model-guided approach.

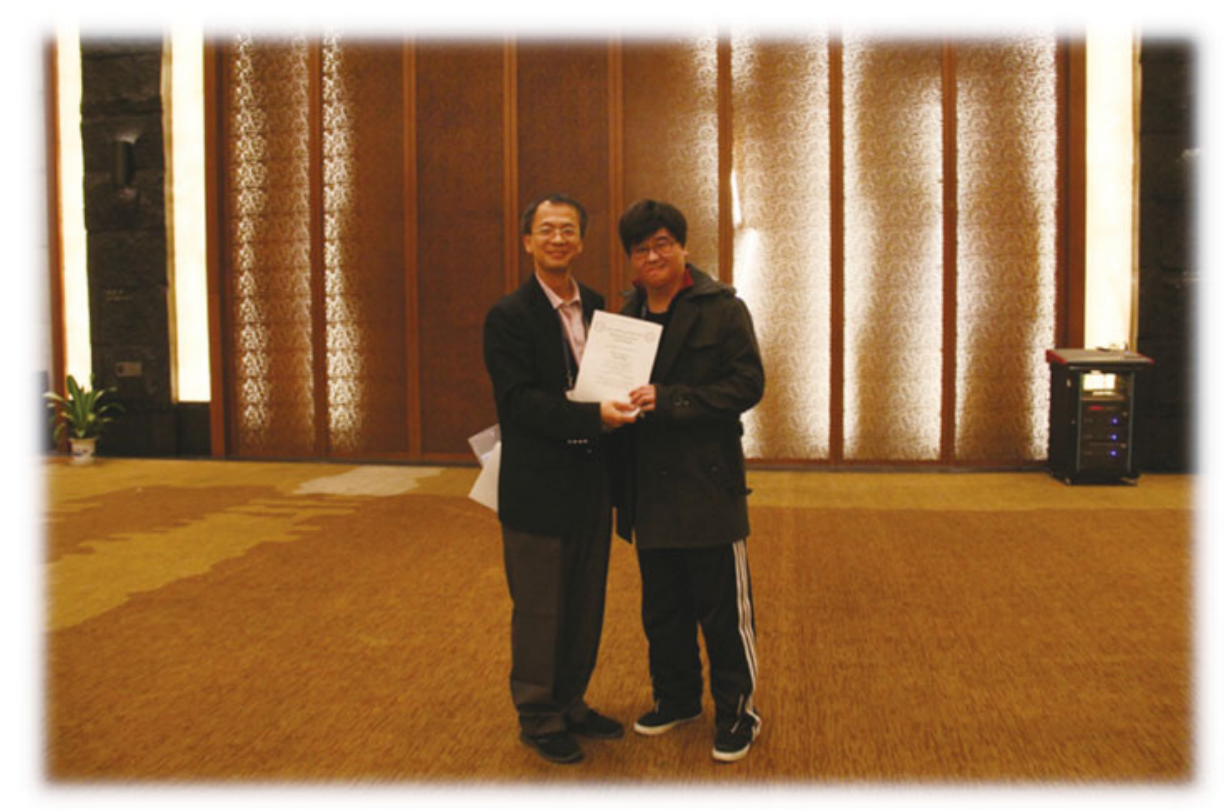

Professor George Guo-Qiang Chen awarded the Gold Medal of poster section to Haoqian Zhang (on the right). 